\title{
Conservative management of single fetal death in twin pregnancy at a tertiary health institution in southern Nigeria: a case report
}

\author{
${ }^{1}$ Babah O A, ${ }^{2}$ Olamijulo A, ${ }^{3}$ Ayanbode O S, ${ }^{4}$ Sanusi M M \\ ${ }_{1,2,3,4}$ Department of Obstetrics and Gynaecology, Lagos University Teaching Hospital, PMB 12003, Idiaraba, \\ Surulere, Lagos, Nigeria
}

\begin{abstract}
:
Introduction: The death of a twin in the second or third trimester of pregnancy is a rare obstetric complication. This might pose management challenge to the obstetrician. It is a cause of great concern and psychological stress to the parents. There are potential complications to the mother and the surviving twin. Despite the fact that Nigeria has one of the highest twinning rates in the world, very few case reports on twinning and its complications exist in the literature.

Case: Presented is a 33 year old, unbooked, primigravid Nigerian woman who had intrauterine fetal death of the second twin at 32 weeks gestation. She was managed conservatively with close fetal surveillance and serial assessment of her clotting profile. She had successful induction of labour at 37 weeks with delivery of a live female baby whose birth weight was $2.45 \mathrm{~kg}$. The dead second twin (foetus papyraceous) was embedded within the placenta.
\end{abstract}

Discussion: Few cases have been reported in other parts of the world. The mode of delivery in most cases being caesarean section. Our patient had successful vaginal delivery following induction of labour with no untoward effect to both mother and surviving twin.

Conclusion: Vaginal delivery is safe following death of a twin and should be considered especially in Africa where there is aversion for caesarean delivery.

Key words: conservative management, dead twin, fetus papyraceous.

\section{Introduction}

The incidence of twinning varies from place to place, being as high as $26.0-40.2$ per 1000 deliveries in Nigeria[1,2] and as low as $6.0-9.0$ per 1000 deliveries in South and South-East Asia[3]. Despite the fact that Nigeria has one of the highest twinning rates in the world, very few case reports on twinning and its complications was found on literature search.

The death of a twin in the second and third trimester of pregnancy is a rare obstetric complication. Reported incidence of single fetal death in twin gestations range between $0.5-6.8 \%$ [4]. A much higher incidence was found in Beijing in which 13 out of the 99 cases of twin pregnancies studied had a single intrauterine fetal death, giving a prevalence of $13.1 \%$ [5]. Most of these are monochorionic twin gestation and can occur at any gestational age[4].

The severity of complications following death of a twin is dependent on the chorionicity and the gestational age at which it occured. If it occurs in monochorionic twins, the risk of the survivor twin being exanguinated exists. This complication is less in dichorionic twins. In all cases however, disseminated intravascular coagulopathy (DIC) may occur, hence need for close fetal surveillance. On the other hand, if the fetal demise occurs in the first trimester, the risk of complications arising is much lower than in situations in which it occurs in the second or third trimester because the dead fetus may be fully re-absorbed with no further evidence of twinning or risk of disseminated intravascular coagulopathy and subsequent ultrasonographic examination may reveal a singleton fetus and this is called the "vanishing twin syndrome)". In the second and third trimester however, the death of a twin might pose a considerable challenge to the obstetrician bearing in mind the risk of disseminated intravascular coagulopathy following an intrauterine fetal death. It is also a cause of great concern and psychological stress to the couple.

\section{Case}

The patient was Miss IV, a 33 year old, primigravid Nigerian woman who presented at the accident and emergency section of the Lagos University Teaching Hospital, Idi-araba, Lagos following referral from a general hospital on account of ultrasonic diagnosis of a dead second twin at 31 weeks gestation. She was then 32 weeks pregnant. She had no complaints and a review of her system was essentially normal.

The pregnancy was spontaneously conceived. She booked at the referral general hospital at 16 weeks gestation following a positive qualitative urine and blood HCG. She had obstetric ultrasound scans at 20 and 27 
weeks gestation; results of which showed viable twin gestation. Her blood group was AB rhesus D positive and her genotype was AA.

On examination, she was not febrile, anicteric and not pale. She had bilateral pitting pedal oedema. Her pulse rate was 88 beats per minute and her blood pressure was $110 / 50 \mathrm{mmHg}$. The symphysiofundal height was $31 \mathrm{~cm}$. The leading twin was in longitudinal lie, cephalic presentation and the fetal heart rate was 156 beats per minute. It was difficult appreciating the lie and presentation of the second twin and the fetal heart sound was not heard.

The clinical impression was intrauterine fetal death of the second twin. The patient and her husband were counselled and the line of management duly explained to them. She was admitted for observation and for conservative management. On admission, a repeat ultrasound scan was done to ascertain the chorionicity, estimated weight and biophysical profile of the surviving twin. The findings were a viable leading twin in cephalic presentation with good cardiac activity and an estimated weight of $1.381 \mathrm{~kg}$ at 30 weeks gestation with a biophysical profile of 10/10 (non-stress test inclusive) while the second twin was devoid of cardiac activity and in breech presentation with collapse of the foetal head and abdomen making the biometric parameters difficult to measure however the femur length measured $45 \mathrm{~mm}$ corresponding to 25 weeks gestation. There was difficulty ascertaining the chorionicity.

She also had a full blood count which showed haematocrit of $34 \%$, normal white blood cells and differentials and a normal platelet count of $245 \times 10^{9} / \mathrm{L}$. Crude bedside clotting time on admission was 3 minutes 30 seconds. Her clotting profile was normal with an international normalized ratio (INR) of 1.00. She was negative for Human Immunodeficiency Virus (HIV) I and II. Other investigations done were serum electrolytes, urea and creatinine, urinalysis and urine microscopy, culture and sensitivity, Veneral Disease Research Laboratory (VDRL) test and 50grammes Glucose Challenge Test (GCT); the results of which were all normal.

On admission, she had antenatal steroid (Dexamethasone) to facilitate lung maturity. She was also maintained on routine haematinics all through. While on admission, she had serial assessment with twice weekly full blood count, clotting profile and biophysical profile. She also maintained a daily fetal kick chart. Two pints of blood was grouped and saved for her. The neonatologists and anaesthetists were informed in case there is need for urgent delivery. Her condition remained satisfactory with good haematological profile and foetal surveillance results. She was scheduled for induction of labour at term.

At 37 completed weeks (after being on admission for 33 days), she had a successful induction of labour with intravaginal prostaglandins (misoprostol) for cervical ripening followed by synchronous artificial rupture of membrane (ARM) and oxytocin infusion. She had continuous cardiotocographic (CTG) monitoring all through labour. She had spontaneous vaginal delivery of a live female baby who appeared morphologically normal with a birth weight of $2.45 \mathrm{~kg}$ and an APGAR score of 9 at 1 minute and 10 at 5 minutes. A fetus papyraceous was noted within the placenta which appeared to be dichorionic as a separating membrane was noted. 


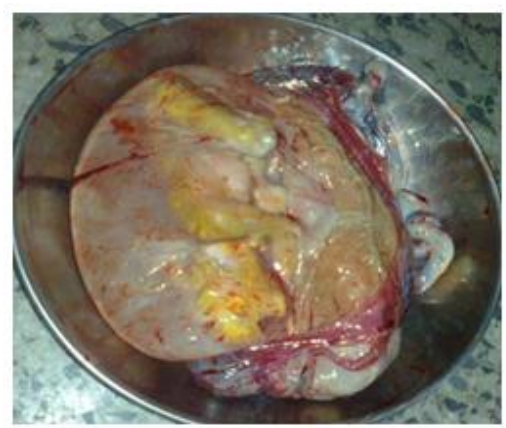

Fig. 1 Placentae with compressed foetus papyraceous within the membranes

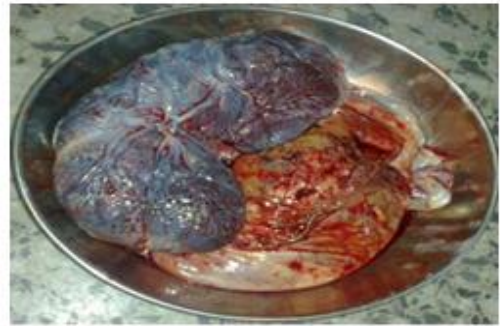

Fig 2 Foetal surface of placentae

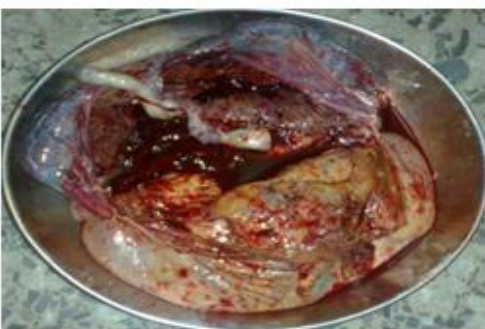

Fig 3 Maternal surface of placentae

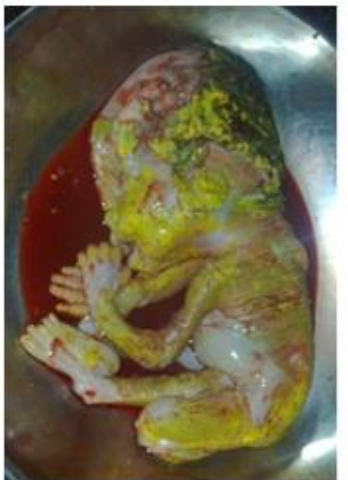

Fig 4 Compressed dead foetus (dissected out from the placenta)

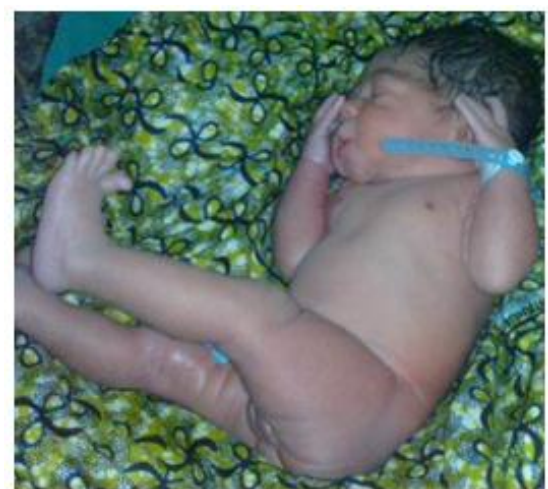

Fig 5 Survivortwin, birth weight $2.45 \mathrm{~kg}$

The placenta and fetus papyraceous were sent to the morbid anatomy department for autopsy and histopathological examination. The live born was handed over to the neonatologists who were in attendance at delivery for neonatal resuscitation and further assessment and post-natal care of the newborn. The inductiondelivery interval was 6 hours 10 mins.

The patient had analgesics and haematinics post-partum. A repeat full blood count done on the second day post-delivery was essentially normal. She was subsequently discharged home with her baby in good condition with a 6 weeks appointment to come for post-natal check. She was counselled prior to discharge to maintain all appointments at the neonatal unit and to ensure her baby was fully immunized.

\section{Discussion}

From literature review, only one case of single fetal death of a twin had been reported in Nigeria despite the high incidence of twinning. This was a case of fetus papyraceous incidentally discovered during routine examination of the placenta and membranes after spontaneous vaginal delivery of a low birth weight, but otherwise healthy infant, to a primigravid mother[6]. Here, we present the management of a case of single fetal death of a twin which was discovered during pregnancy and subsequently managed conservatively for 33 more days to improve the chance of fetal survival and then delivered vaginally at term following a successful induction of labour. Similar cases had been reported in other parts of the world which were also managed conservatively until delivery[7-10].

Death of a twin poses a management challenge to the obstetrician and has medicolegal implications hence care must be taken when making a decision on whether or not to offer conservative management. 
However, it is important that the mode of treatment offered be individualised and the risks of conservative management should be weighed against the risk of preterm birth. Where a decision is taken to embark on conservative management, then close monitoring of both mother and the fetus must be performed. The patient presented had serial assessment of her clotting profile and serial full blood count including absolute platelet count was done because of the risk of disseminated intravavscular coagulopathy (DIC) that may follow in-utero fetal death.

The death of a twin normally puts the second twin at risk of dying especially in cases of monochorionic placentation. However, it must also be borne in mind that even when it is a dichorionic placentation, microvascular connections between the two placentae cannot be completely ruled out, hence close fetal surveillance must be instituted if conservative management is to be considered. Our patient had twice weekly biophysical profile and daily fetal kick chart to monitor the wellbeing of the survivor twin.

Other investigations that could be done include fetal blood sampling which may be a useful diagnostic tool in identifying those fetuses that are not anaemic and hence unlikely to be at risk of developing a cerebral lesion especially in cases in which twin-twin transfusion syndrome is suspected[11].

There is no specific contraindication to vaginal delivery following death of a twin. However, situations may arise sometimes in which caesarean section may be indicated and the rate varies considerably ranging from $19-92 \%$ [12]. In a number of cases reported, delivery was by caesarean section, the indication in most cases being fetal heart rate irregularities[8,9,10]. Cord abnormality (commonly torsion of the umbilical cord) was observed in some of these fetuses and was thought to have caused intrauterine hypoperfusion resulting in the death of a twin. There was no cord abnormality observed in the case presented.

The surviving co-twin however may suffer from some form of neurological damage even when findings on close fetal surveillance has been normal, hence it is adviseable to carry out a thorough neonatal evaluation in the surviving twin to detect abnormalities in the renal, circulatory and central nervous systems[12].

Psychological support for the patient and her partner is very important because death of a twin is a cause of great anxiety to the patient and her family. The patient remains under constant threat of the possibility of the survivor twin dying. Our patient and her husband were counselled from time to time until delivery and discharge to allay their anxiety and fears.

\section{Conclusion}

It is worth-while employing conservative management following death of a twin. However, this preferably should be in a tertiary institution and a multidisciplinary approach should be employed. Close fetal surveillance and serial monitoring of the patient's haematological profile should be performed during conservative management. The risk of offering conservative management should however be weighed against the risk of a preterm birth and its sequelae. Vaginal delivery is safe and should be considered especially in Africa where there is aversion for caesarean delivery due to the socio-cultural beliefs.

Conflict of interest: The author hereby declares that there is no conflicting interest.

\section{Contributions of authors:}

OA: consultant who supervised the management of the patient and preparation of this manuscript.

BOA: senior registrar who was involved in the management of the patient, supervised the induction of labour, took the delivery and prepared this manuscript.

AOS: junior registrar who participated in the management of this patient, monitored the induction of labour and assisted during the delivery.

SMM: junior registrar who participated in the management of this patient.

\section{References}

[1] Abasiattai AM, Umoiyoho AJ, Utuk NM, Shittu DG. Incidence and mode of delivery of twin pregnancies in Uyo, Nigeria. Niger Med J. 2010; 51: $170-2$.

[2] Akinboro A, Azeez MA, Bakare AA. Frequency of twinning in south-west Nigeria. Indian Journal of Human Genetics. 2008; 14(2): $41-47$.

[3] Smits J, Monden C. Twinning across the developing world. PLOS ONE. 2011; 6(9): e25239.

[4] Kursheed R, Ahmed A, Parveen K. Foetus papyraceous in twin pregnancy - A case report. The Internet Journal of Gynaecology and Obstetrics. 2009;11(2).

[5] Juntao L, Jiaxin Y, Xuming B, Yu Z. Conservative management of twin pregnancy with single fetal death. Clin Med Sci J. 2000; 15(2): $103-6$.

[6] Airede LR, Ahmed Y. Fetus papyraceous: A case report. Annals of African Medicine. 2005; 4(3): 136 - 138.

[7] Chen CD, Ko TM, Hsieh FJ, Huang SF. Intrauterine death of co-twin in the third trimester: a case report of twin to twin transfusion syndrome and cord accident. J Formos Med Assoc. 1993; 92(7): 665 - 7.

[8] Preis K, Ciach K, Kowalewska-Wlas A, Wójcik D, Swiatkowska-Freundi M, Klasa-Mazurkiewicz D. Twin pregnancy with single fetal death due to triploidy - a case report. Ginekol Pol. 2005; 76(8): 648 - 51. 
[9] Fleisch MC, Hoehn T. Intrauterine fetal death after multiple umbilical cord torsion - complication of a twin pregnancy following assisted reproduction. J Assist Reprod Genet.2008; 25(6): 277 - 279.

[10] Athwal S, Mallard K, Lakhoo K. Twin reversed arterial perfusion (TRAP) sequence in association with VACTERL association: a case report. Journalof Medical Case Reports. 2010; 4: 411.

[11] Senat MV, Bernard JP, Loizeau S, Ville Y. Management of single fetal death in twin to twin transfusion syndrome: a role for fetal blood sampling. Ultrasound Obstet Gynecol. 2002; 20(4): $360-3$.

[12] Woo HHN, Sin SY, Tang LCH. Single fetal death in twin pregnancies: review of the maternal and neonatal outcomes and management. HKMJ. 2000; 6(3): 293 - 300. 